
\title{
28 Research Square \\ Echocardiographic and Clinical Features in Diagnosis of Rare Cardiac Myxomas
}

qi yanhua

The Second Affiliated Hospital of Xi'an Jiaotong University https://orcid.org/0000-0002-1332-5221

miaoyan Ma

Xian Jiaotong University: Xi'an Jiaotong University

\section{Yajuan Wei}

Xian Jiaotong University: Xi'an Jiaotong University

jinfang wu

Xian Jiaotong University: Xi'an Jiaotong University

peirong gao

Xian Jiaotong University: Xi'an Jiaotong University

xiaopeng li ( $\square$ xiaopengli8@126.com )

Xian Jiaotong University: Xi'an Jiaotong University

baomin liu

Xian Jiaotong University: Xi'an Jiaotong University

\section{Research article}

Keywords: Cardiac myxoma, Echocardiographic, Diagnosis

Posted Date: March 5th, 2021

DOI: https://doi.org/10.21203/rs.3.rs-263887/v1

License: (c) (1) This work is licensed under a Creative Commons Attribution 4.0 International License.

Read Full License 


\section{Abstract}

Background: Cardiac myxoma(CM) is a rare cardiac disease, with the majority located in the left atrium and less commonly in the right atrium, right ventricular, biatrial, left ventricular, right ventricular outflow tract or heart valves. These rare CMs need to be distinguished from other masses. The purses of our study is to provide important value in finding the extra-atrial tumor and making the correct diagnosis by reviewed echocardiographic and clinical features of five rare CMs.

Methods: We reviewed medical records and echocardiographic data in all patients with surgery diagnosis of rare CMs from January 2019 to June 2020 in the second affiliated hospital, Xi'an Jiaotong University.

Results: Five patients were enrolled in our study. The sites of CMs were rare and located separately on RV, PV, LV, LA and LVOT, LA. Four cases was myxomas and one was lipoma. Five patients recovered well after operation.

Conclusions: Cardiac tumors developed in unusual locations should be considered to exclude the possibility of myxoma.

Subheadings: Echocardiographic of rare Cardiac Myxomas

\section{Background}

Cardiac myxoma(CM) is a rare cardiac disease with an overall incidence of about $0.5-1.0 / \mathrm{million} / \mathrm{y}^{[1]}$. CM accounts for approximately $70 \%$ of the adult cardiac tumors, with the majority $(75 \%)$ located in the left atrium and less commonly in the right atrium $(20 \%)^{[2]}$. Rarely CMs may be present in right ventricular (RV), biatrial, left ventricular (LV) ${ }^{[3]}$, right ventricular outflow tract (RVOT) ${ }^{[4]}$ or heart valves ${ }^{[5,6]}$. The sporadic type accounts for about $93 \%$, and the multiple type present in familial forms ${ }^{[7]}$. These rare CMs need to be distinguished from other masses, such as papillary fibroelastoma, rhabdomyoma, lipoma and thrombi ${ }^{[8]}$. Echocardiographic and clinical features may provide very important information. We reported five rare site of myxoma-like masses, especially pulmonary valve (PV) myxoma was never been reported before. Therefore, our study could provide important value in finding the extra-atrial tumor and making the correct diagnosis.

\section{Methods}

We reviewed medical records and echocardiographic data in all patients with surgery diagnosis of rare CMs from January 2019 to June 2020 in the second affiliated hospital, Xi'an Jiaotong University. The written informed consent was waived because this study was retrospective and anonymous clinical data were used for analysis. We followed the Helsinki Declaration Principles to conduct this study.

We evaluated the demographic data including age, sex, cardiovascular risk factors, past history of stroke or other systemic embolic events and final pathological diagnosis and postoperative recurrence, and so 
on.

Echocardiographic features of CMs were recorded, including tumor size (width and height), shape (round vs. ovoid), irregularity of the surface (smooth vs. polypoid), presence of pedicle (narrow stalk vs. broad base) and mobility.

\section{Results}

Five patients were enrolled in our study, which transthoracic echocardiography (TTE) identified as CMs. Finally, the result was confirmed by surgical resection and pathology. The echocardiographic findings of rare CMs were summarized in Table 1 , and the clinical and laboratory characteristics were summarized in Table 2. The sites of CMs were rare and located separately on RV(Fig. 1), PV(Fig. 2), LV(Fig. 3, video2), LA and LVOT(Fig. 4), LA(Fig. 5). Four myxomas were solitary, one was multiple.

Table 1

Echocardiographic characteristics of the rare $\mathrm{CMs}$

\begin{tabular}{|c|c|c|c|c|c|}
\hline & Case1 & Case2 & Case3 & Case4 & Case5 \\
\hline Location & RV & PV & LV & $\begin{array}{l}\text { LA and } \\
\text { LVOT }\end{array}$ & LA \\
\hline \multirow{2}{*}{$\begin{array}{l}\text { Size(width*height } \\
\mathrm{mm} \text { ) }\end{array}$} & \multirow[t]{2}{*}{$21 * 16$} & \multirow[t]{2}{*}{$14 * 10$} & \multirow[t]{2}{*}{$22 * 15$} & $34 * 26$ & \multirow[t]{2}{*}{$77 * 45$} \\
\hline & & & & $18 * 16$ & \\
\hline Shape(round,ovoid) & round & round & irregular & ovoid & ovoid \\
\hline $\begin{array}{l}\text { Surface } \\
\text { (polypoid,smooth) }\end{array}$ & smooth & smooth & polypoid & polypoid & smooth \\
\hline $\begin{array}{l}\text { Stalk (narrow } \\
\text { stalk,broad base) }\end{array}$ & broad base & narrow stalk & $\begin{array}{l}\text { narrow } \\
\text { stalk }\end{array}$ & $\begin{array}{l}\text { narrow } \\
\text { stalk }\end{array}$ & $\begin{array}{l}\text { broad } \\
\text { base }\end{array}$ \\
\hline Mobile or non-mobile & non-mobile & mobile & mobile & mobile & mobile \\
\hline Ultrasonic diagnosis & myxoma & $\begin{array}{l}\text { myxoma/ } \\
\text { leiomyoma }\end{array}$ & myxoma & myxoma & myxoma \\
\hline Other abnormality & $\begin{array}{l}\text { patent foramen } \\
\text { ovale }(3 \mathrm{~mm})\end{array}$ & / & / & l & / \\
\hline Pathology & myxoma & myxoma & lipoma & myxoma & myxoma \\
\hline
\end{tabular}


Table 2

Clinical and laboratory characteristics of the patients with rare CMs

\begin{tabular}{|llllll|}
\hline & Case1 & Case2 & Case3 & Case4 & Case5 \\
\hline Age(years) & 17 & 57 & 77 & 52 & 66 \\
\hline Sex & Male & Male & Female & Male & Female \\
\hline Incidentally found myxoma & Yes & Yes & Yes & Yes & Yes \\
\hline Past clinical history & $/$ & $/$ & Yes & $/$ & $/$ \\
Hypertension & $/$ & $/$ & $/$ & $/$ & $/$ \\
Diabetes & $/$ & $/$ & $/$ & $/$ & $/$ \\
Dyslipidemia & $/$ & $/$ & $/$ & $/$ & $/$ \\
History of systemic embolism & & & & & \\
\hline Cardiac symptoms & $/$ & $/$ & $/$ & $/$ & Yes \\
Dyspnea & $/$ & $/$ & Yes & $/$ & Yes \\
Chest pain & Yes & $/$ & $/$ & $/$ & $/$ \\
Palpitation & $/$ & $/$ & $/$ & Yes & Yes \\
Dizziness/syncope & $/$ & Yes & $/$ & $/$ & $/$ \\
Cough & & & & & $/$ \\
\hline Embolic phenomenon & $/$ & $/$ & Yes & Yes & $/$ \\
Brain & $/$ & $/$ & $/$ & $/$ & $/$ \\
Coronary & $/$ & $/$ & $/$ & $/$ & $/$ \\
Lower extremity & & & & & \\
Pulmonary artery & & & & & $/$ \\
\hline
\end{tabular}

Five patients recovered well after operation, and there were no complications during follow-up, no postoperative AF developed, no or mild regurgitation of the corresponding valve, no postoperative recurrence.

\section{Discussion}

CMs were the most frequent primary benign cardiac tumors in adults accounting for $50-85 \%$. LA is the well-known, most prevalent site of myxomas with the incidence of $77-95 \%$ and $80 \%$ of which are attached to the interatrial septum. Tumors other than myxomas found in the LA cavity might be confused with myxomas and myxomas involving the sites other than the LA may be mistaken for other tumors. CMs are attached to the endocardium. There are two types of CMs: (1) round type, characterized by solid, 
round, regular shape and nonmobile surface; (2) polypoid type, characterized by soft, irregular shape and mobile surface. The polypoid type of tumor is associated with a higher occurrence of systemic embolism than the round type ${ }^{[9]}$.

TTE is a widely available, noninvasive diagnostic method for initial evaluation of cardiac myxomas, with high sensitivity and specificity. Its accuracy can approach $95 \%{ }^{[10]}$. It can usually provide adequate diagnostic information, such as location, attachment site, size, shape, mobility, morphology and hemodynamic consequences of the tumor.

Our study reported five rare sites of CMs diagnosed by TTE. The tumors at these sites should be distinguished from thrombi, papillary fibroelastoma(PFEs), leiomyoma, rhabdomyoma and lipoma. Some research reported that non-myxoma tumors were significantly smaller than myxomas ${ }^{[11]}$. Thrombi had comparable or larger size than myxomas. Importantly, in patients with AF or myocardial infarction, the diagnosis of thrombus should be considered primarily, even if it mimics myxoma. If a relatively small tumor is found outside the atrium, the possibility of myxoma should be considered after the exclusion of thrombus by history. However, it is very difficult to distinguish leiomyoma, lipoma, and myxoma by echocardiography, and in our case, one lipoma was misdiagnosed.

PFEs, which are mainly found in the valves, are rare benign tumors that can cause embolism. Cardiac valves were predominantly involved (76.5\%); the most commonly valve affected was the aortic valve (29.4\%), followed by the mitral valve (17.6\%). Concurrent valvular disease was observed in $41.7 \%$ of patients. PFE is usually small, appears singly, and can cause potentially serious complications ${ }^{[12]}$. However, a tumor on the valve cannot be completely rule out as myxoma. Yuan $\mathrm{S}^{[13]}$ reported a 47-yearold male with a cardiac mass arising from the tricuspid valve, which was misdiagnosed as a cystic myxoma. The mass turned out to be tricuspid cystic myxomatus degeneration with a primary cardiacleiomyoma finally. To our best of our knowledge, the pulmonary valve myxomas in our study have not been reported.

Giant stalkless myxomas had scarcely ever been reported in the literature ${ }^{[14]}$. Such myxomas are often immobile and require a more extensive surgical resection. Therefore, echocardiography and other multimodality imaging can be helpful in diagnosis and operation planning ${ }^{[15]}$. Case 5 in our study was a giant left atrial myxoma. Part of the left atrium was excised and repaired in operation. Nevertheless, a stalkless myxoma can result in low output state considering that it is a space-occupying lesion which is decreasing ventricular filling. In our case, decreasing ventricular filling can cause symptoms such as dizziness or syncope.

The clinical signs, manifestations, and symptoms produced are non-specific and determined by the location, size, and mobility of tumor ${ }^{[16]}$. There are 3 classic presentation for patients with CMs ("myxoma triad"): hemodynamic consequences, systemic embolism and constitutional or systemic manifestations. Smooth tumors are larger, occur with or without obstructive symptoms, whereas villous myxomas entailed a high embolic risk. This highlights the importance of the echocardiogram in describing the 
morphologic characteristics of these tumors, which predicts patients at higher risk of having an embolic complication and those who may require surgery as soon as possible after diagnosis. Even small and

nonmobile cardiac myxomas with a round regular shape may cause recurrent cerebral infarction ${ }^{[17]}$.

\section{Conclusions}

CMs are the most frequently diagnosed primary cardiac tumor. They have characteristic imaging features that may frequently suggest the diagnosis and aid in differentiating these lesions from other intracardiac masses. This study described clinical and echocardiographic characteristics of 5 patients with echocardiographic diagnosis of rare cardiac myxomas. Cardiac tumors developed in unusual locations should be considered to exclude the possibility of myxoma.

\section{List Of Abbreviations}

CM Cardiac myxoma

RV Right ventricular

LV Left ventricular

RVOT Right ventricular outflow tract

PV Pulmonary valve

TTE Transthoracic echocardiography

PFEs Papillary fibroelastoma

\section{Declarations}

Ethics approval and consent to participate The written informed consent was waived because this study was retrospective and anonymous clinical data were used for analysis. We followed the Helsinki Declaration Principles to conduct this study.

Consent for publication Not applicable

Availability of data and materials All data generated during this study are included in this published article.

Competing interests The authors declare that they have no competing interests.

Funding None

Authors' contributions 
Yanhua Qi Yanjuan Wei, Miaoyan Ma, and Peirong Gao are responsible for the conception and design of the study, drafting, and writing of the article. Donggang Han and Xiaopeng Li are responsible for review and editing of the content. Jinfang Wu and Baomin Liu participate in the final approval of the manuscript and agreed to be accountable for all aspects of the work.

Yanhua Qi and Miaoyan Ma contributed equally to this work and should be considered co-first authors.

Xiaopeng Li and Baomin Liu contributed equally to this work and should be considered cocorrespondence authors.

Acknowledgements Not applicable

\section{References}

1. Cianciulli TF, Cozzarin A, Soumoulou JB, et al. Twenty Years of Clinical Experience with Cardiac Myxomas: Diagnosis, Treatment, and Follow Up. J Cardiovasc Imaging. 2019;27(1):37-47.

2. Fernandes GC, Alejandro Pajares $\mathrm{W}$, Amboss N, et al. Right atrial myxoma with eripheral eosinophilia: Eosinophilia in cardiac myxoma. J Card Surg. 2019;1-4.

3. Yu L, Gu T, Shi E. Echocardiographic Findings and Clinical Correlation With Cardiac Myxoma. JACC: CARDIOVASCULARIMAGING. 2016; 05;95(5).

4. Katiyar G, Vernekar JA, Lawande A, et al. Cardiac MRI in right ventricular outflow tract myxoma: Case report with review of literature. J Cardiol Cases. 2020;223(3):128-31.

5. Fernández AL, Vega M, El-Diasty MM, et al. Myxoma of the aortic valve. Interact Cardiovasc Thorac Surg. 2012;15:560-2.

6. Yoon JH, Kim JH, Sung YJ, et al. Cardiac myxoma originating from the anterior mitral valve leaflet. J Cardiovasc Ultrasound. 2011;19:228-31.

7. Pinede L, Duhaut P, Loire R. Clinical presentation of left atrial cardiac myxoma. Medicine. 2001;80:159-72.

8. Brylie JM, BSc MBBS. Multi Modality Imaging Features of Cardiac Myxoma. J Cardiovasc Imaging. 2020;28(4):235-43.

9. Ha JW, Kang WC, Chung N, et al. Echocardiographic and morphologic characteristics of left atrial myxoma and their relation to systemic embolism. Am J Cardiol. 1999;83(11):1579-82.

10. Karabinis A, Samanidis G, Khoury M, et al. Clinical presentation and treatment of cardiac myxoma in 153 patients. Medicine(Baltimore). 2018;97:37(e12397).

11. Lee SH, Park JS, Park JH, et al. Comparison of Clinical and Echocardiographic Characteristics between Cardiac Myxomas and Masses Mimicking Myxoma. Korean Circ J. 2020;50(9):822-32.

12. Val Bernal JF, Mayorga M, Garijo MF, et al. Cardiac papillary fibroelastoma: retrospective clinicopathologic study of 17 tumors with resection at a single institution and literature review. Pathol Res Pract. 2013;209(4):208-14. 
13. Yuan S, Li J, Zheng Z. Tumor-like tricuspid myxomatus degeneration with a primary cardiacleiomyoma: A rare case report. J Card Surg. 2021;361(1).

14. Theodoropoulos KC, Tountas C, Masoero G, et al. A stalkless myxoma-does it behave better? Hellenic Journal of Cardiology, https:// doi.org/10.1016/j.hjc.2020.11.010.

15. Kawa Haji A, Nasis. Radiological characteristics of atrial myxoma in Cardiac Computed Tomography. Journal of Cardiovascular Computed Tomography. 2017;11:234-6.

16. El Sabbagh A, Al-Hijji MA, Thaden JJ, et al. Cardiac Myxoma The Great Mimicker. JACC: CARDIOVASCULARIMAGING. 2017;10(2):203-6.

17. Saito Y, AizawaY, Monno K, et al. Small, smooth, nonmobile cardiac myxoma detected by transesophageal echocardiography following recurrent cerebral infarction: a case report. J Med Case Rep. 2017;11:131.

\section{Figures}

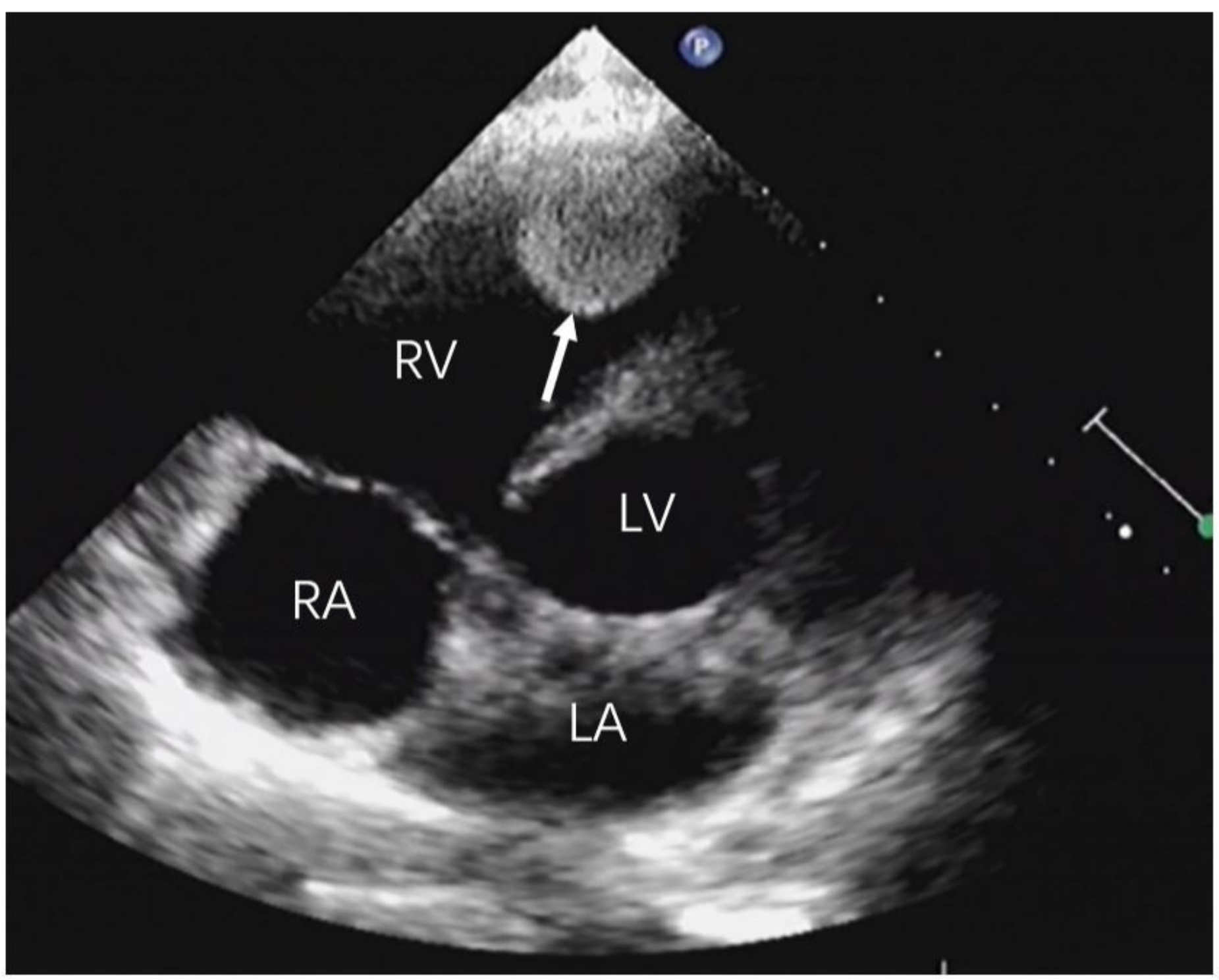




\section{Figure 1}

The sites of CMs were rare and located separately on RV(Fig1),
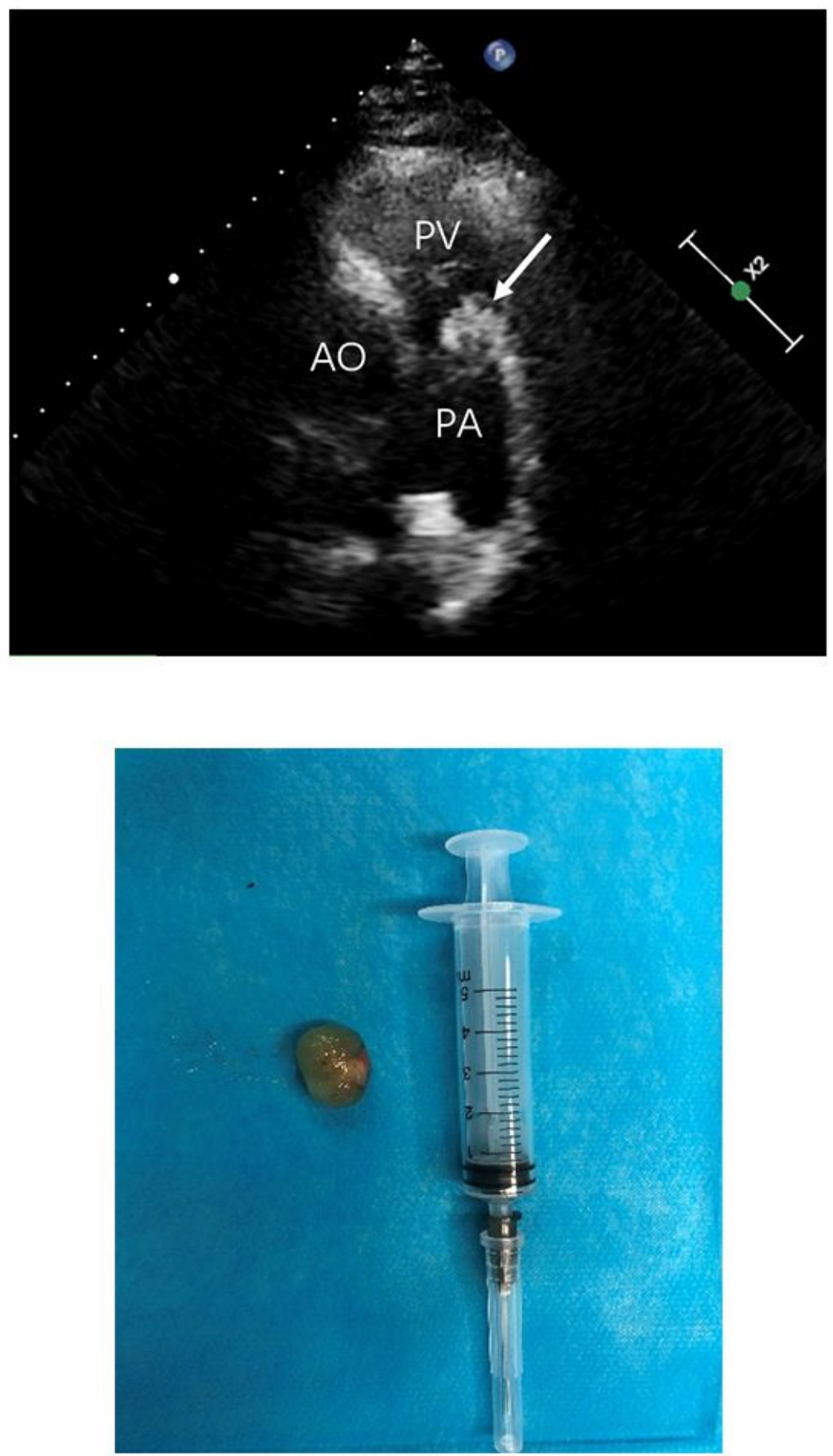

Figure 2

The sites of CMs were rare and located separately on PV(Fig2), 

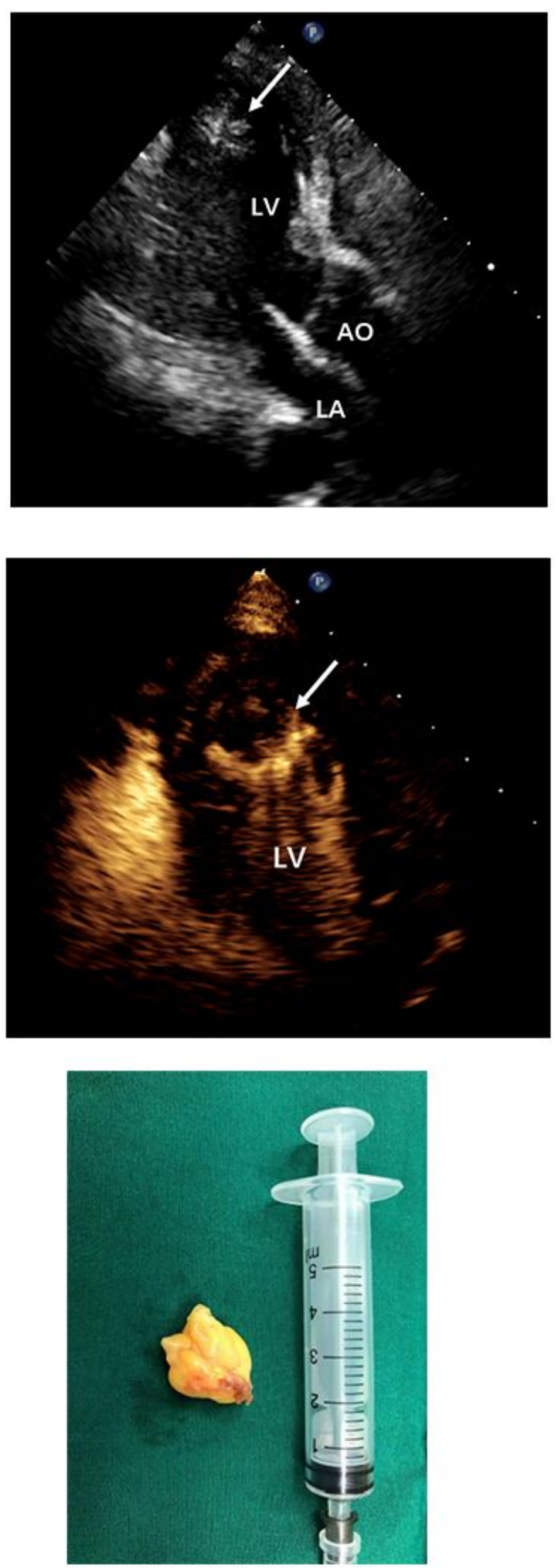

Figure 3

The sites of CMs were rare and located separately on LV(Fig3, video2), 

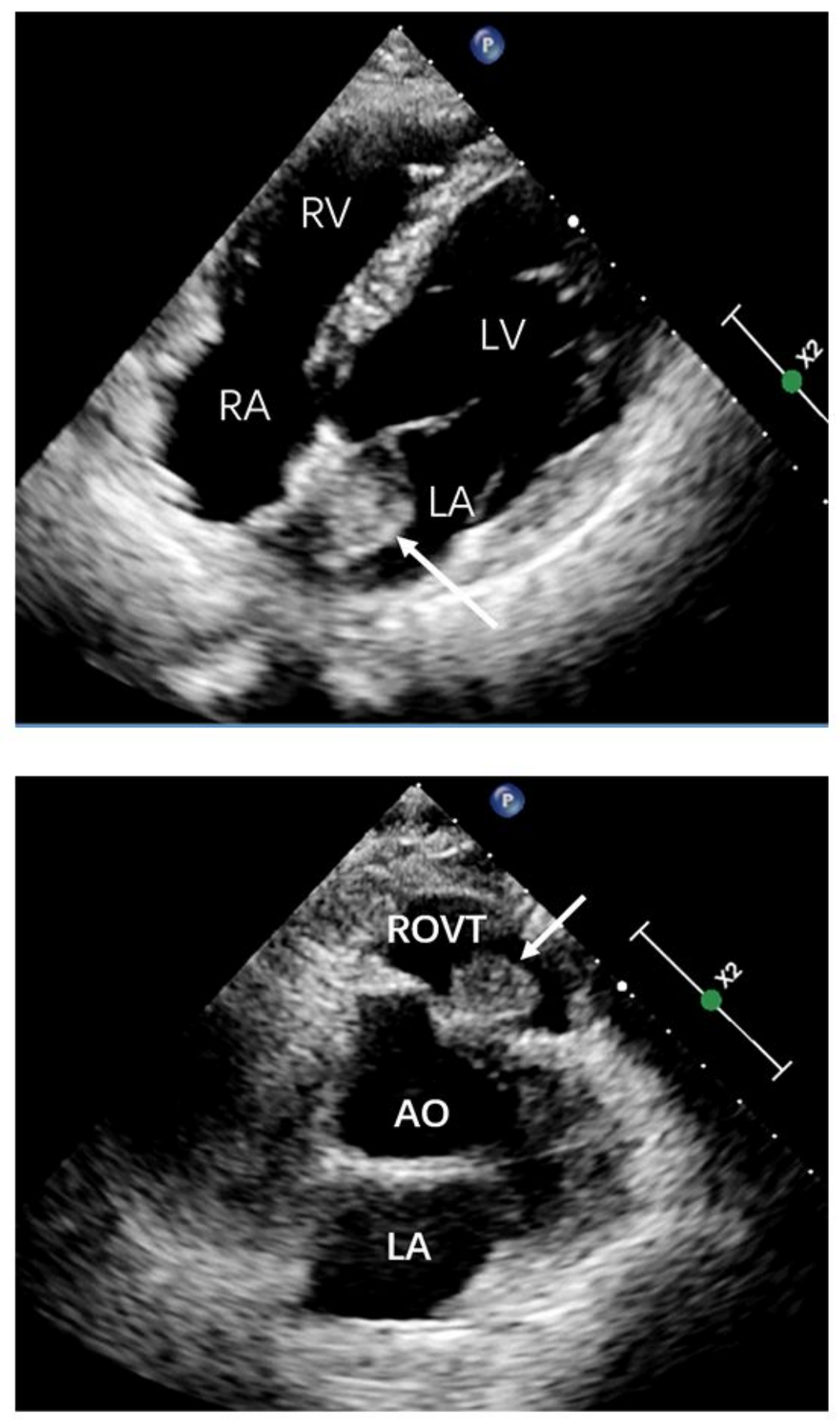

Figure 4

The sites of CMs were rare and located separately on LA and LVOT(Fig4), 


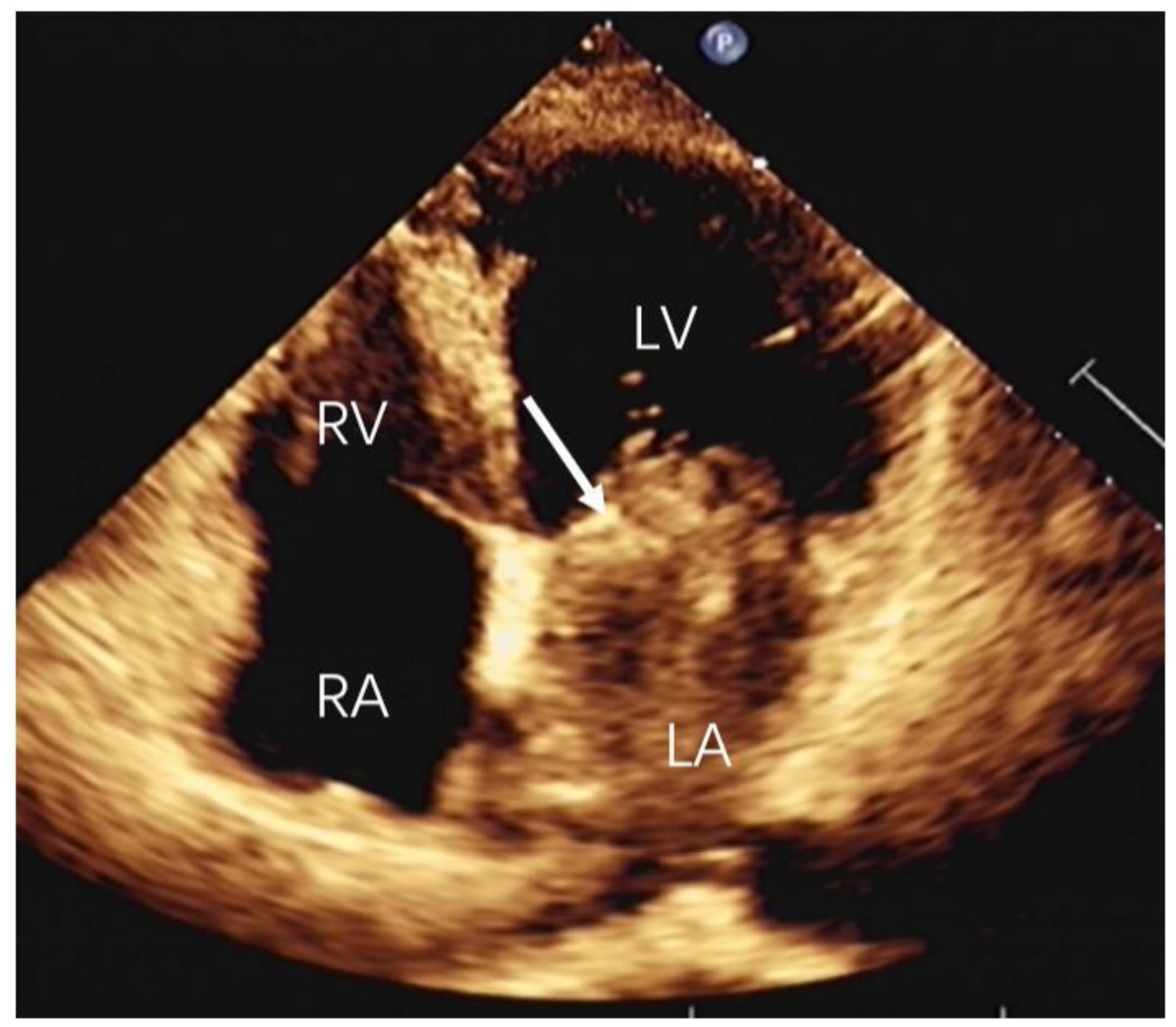

Figure 5

The sites of CMs were rare and located separately on LA(Fig5). 